\title{
Rat Intestine Secretes Discoid High Density Lipoprotein
}

\author{
P. H. R. Green, A. R. Tall, and R. M. Glickman, Gastrointestinal Unit, \\ Beth Israel Hospital, Boston, Massachusetts 02215, Department of Medicine, \\ Boston University School of Medicine, Boston, Massachusetts 02118, \\ and Gastrointestinal Unit, Columbia University College of Physicians \\ and Surgeons, New York 10032
}

\begin{abstract}
A B S T R A C T High density lipoprotein was isolated from pooled rat serum and mesenteric lymph of lymph fistula rats. In most experiments, 5,5'-dithionitrobenzoic acid, an inhibitor of the enzyme lecithin:cholesterol acyltransferase, was added during the collection of lymph to prevent modification of the lipid composition of newly secreted lipoproteins. Negative staining electron microscopy of lymph high density lipoprotein revealed discoidal particles $(190 \pm 3 \times 55 \pm 1 \AA)$ which tended to form rouleaux, smaller spherical particles were also present. Serum high density lipoprotein contained only spherical particles (diameter $93 \pm 4 \AA$ ). Lipid analysis showed that lymph high density lipoprotein was enriched in phospholipid and deficient in cholesterol esters when compared to serum high density lipoprotein. The phospholipid to cholesterol esters ratio was greatest in basal lymph high density lipoprotein when compared to fatty lymph and serum high density lipoprotein. From analysis of the lipid compositional data and direct particle measurement by electron microscopy, it could be determined that $\cong 50 \%$ of basal lymph high density lipoprotein and $30 \%$ of fatty lymph high density lipoprotein was discoid. Basal lymph high density lipoprotein was enriched in apoA-I and deficient in the arginine-rich peptide, and the apoprotein composition of fatty lymph high density lipoprotein more closely resembled serum. These observations demonstrate that intestinal lymph contains two types of high density
\end{abstract}

Reprint requests should be addressed to Dr. Glickman, Dept. of Medicine, Gastrointestinal Unit, Columbia University College of Physicians and Surgeons, New York.

Received for publication 6 September 1977 and in revised form 16 November 1977. lipoprotein particles, a discoid nascent particle deficient in cholesterol ester and rich in apoA-I, and spherical high density lipoprotein derived from plasma. A significant amount of lymph high density lipoprotein appears to be secreted by the intestine.

\section{INTRODUCTION}

Although the role of the intestine in the synthesis and secretion of chylomicrons and very low density lipoproteins is well established $(1,2)$, less is known concerning intestinal high density lipoprotein (HDL) ${ }^{1}$ metabolism. Previous studies, which demonstrated incorporation of radioactive amino acids into plasma HDL in hepatectomized animals, suggested that the intestine may synthesize HDL (3). Windmueller and Spaeth (4), using an isolated perfused intestinal preparation in rats, demonstrated radioactive amino acid incorporation into HDL apoproteins in lymph and perfusate. Studies from this laboratory have shown that apoA-I, the principal apoprotein of plasma HDL is the major apoprotein of mesenteric lymph chylomicrons (5) and is actively synthesized by rat small intestine during lipid absorption. These studies also demonstrated that in the fasting state, $85 \%$ of lymph apoA-I was present in the $d>1.006$ $\mathrm{g} / \mathrm{ml}$ infranate suggesting that this apoprotein was associated with mesenteric lymph HDL, and raises the possibility that this HDL might be of intestinal origin.

While HDL is present in mesenteric lymph (6), it has been assumed in part to represent filtration of HDL

${ }^{1}$ Abbreviations used in this paper: ARP, arginine-rich protein; DTNB, 5,5'-dithionitrobenzoic acid; HDL, high density lipoprotein; LCAT, lecithin:cholesterol acyltransferase; PL, phospholipid. 
from the circulation into lymph (7). The recent report (8) that HDL are secreted from perfused rat liver as discoidal bilayer particles raises the possibility that nascent HDL from other tissues may be secreted in a similar form.

The present studies were undertaken to determine whether mesenteric lymph contains nascent HDL and to compare these particles with serum HDL. Since mesenteric lymph contains lecithin:cholesterol acyltransferase (LCAT) (9), an inhibitor of this enzyme was added to lymph. Under these conditions, mesenteric lymph was found to contain discoidal HDL which was enriched in apoA-I and deficient in cholesterol ester. These results are a direct demonstration that a major portion of mesenteric lymph HDL are not filtered from plasma but are secreted from the intestine as phospholipid apoprotein bilayer disks.

\section{METHODS}

Lipoprotein preparation. The main mesenteric lymphatic and duodenum of 200-g male Sprague-Dawley rats (Charles River Breeding Laboratories, Wilmington, Mass.) were cannulated, as previously described (1). Animals were placed in restraining cages and allowed free access to $5 \%$ dextrose in $0.9 \%$ saline. Lymph was collected with and without anticoagulation on ice over a 12 -h period. $5,5^{\prime}$-dithionitrobenzoic acid (DTNB) in $0.75 \mathrm{M}$ phosphate buffer, $\mathrm{pH} 7.4$, was added during collection to a final concentration of $1 \mathrm{mM}$ to inhibit the action of LCAT (8). Fatty lymph was obtained after the intraduodenal infusion of Intralipid (Cutter Laboratories, Inc., Berkeley, Calif.) and was collected without the addition of DTNB. Both fasting and fatty lymph were defibrinated and the HDL fraction was prepared by ultracentrifugation at $4^{\circ} \mathrm{C}$ in the $50.2 \mathrm{~T}$ rotor of a Beckman preparative ultracentrifuge (Beckman Instruments, Inc., Palo Alto, Calif.). Lipoprotein separations from basal, fatty lymph, and serum were performed in parallel. The density of lymph or serum was increased by the addition of salt solutions containing $\mathrm{NaCl} / \mathrm{KBr}$ and $0.04 \%$ disodium EDTA. HDL was isolated between densities 1.075 and 1.21 $\mathrm{g} / \mathrm{ml}$ and then separated from the subnatant by aspiration. HDL was purified by recentrifugation at the upper density limit. Centrifugation was at $35,000 \mathrm{rpm}$ for $44 \mathrm{~h}$. Lipoprotein fractions were dialized for $24-48 \mathrm{~h}$ at $4^{\circ} \mathrm{C}$ against $0.9 \%$ saline containing $0.04 \%$ EDTA, pH 7.4, and then concentrated by vacuum dialysis to a protein concentration of $2-3 \mathrm{mg} / \mathrm{ml}$. Serum HDL was prepared from pooled rat serum by ultracentrifugation in the presence of DTNB added to a final concentration of $1 \mathrm{mM}$.

Electron microscopy. Lymph and plasma HDL fractions were negatively stained with $2 \%$ sodium phospho-tungstate, pH 7.4, on Formvar-coated copper grids (Monsanto Co., St. Louis, Mo.). Electron micrographs were obtained with an AEI-6B electron microscope, calibrated with a catalase standard, at $\cong \times 100,000$. Each sample was examined on three different grids. Five photographs of each grid were randomly taken from areas of the grid where particles were not obviously confluent. Particles were sized from the photographs by measuring the diameters of all particles present in a square measured in the center of each photograph.

Polyacrylamide gel electrophoresis. Isolated lipoprotein subfractions were delipidated in ethanol-ether $(3: 2, \mathrm{vol} / \mathrm{vol})$ at $4^{\circ} \mathrm{C}$ as described by Brown et al. (10), and the apoproteins subjected to sodium dodecyl sulfate polyacrylamide gel electrophoresis. The apoprotein bands were stained with Coomassie Blue and quantitated densitometrically (1).

Lipid analysis. Lipids were extracted from lymph and serum lipoproteins in chloroform/methanol $(2: 1, \mathrm{vol} / \mathrm{vol})$ according to the method of Folch et al. (11). Lipid composition was determined by quantitative thin-layer chromatography by the method of Downing (12) as modified by Katz et al. (13). Cholesterol, cholesterol ester, triacylglycerol, and fatty acid were separated in the solvent system of hexane, diethyl ether, and acetic acid (70:30:1, vol/vol). Individual phospholipids were developed in chloroform, methanol, water, and acetic acid $(65: 25: 4: 1, \mathrm{vol} / \mathrm{vol})$. Protein determinations were performed according to the method of Lowry et al. (14).

\section{RESULTS}

Electron microscopy. HDL was prepared from pooled serum and mesenteric lymph and examined by negative staining. Negative stain electron microscopy of serum HDL $(n=5)$ (Fig. la) showed a uniform spherical population of particles with a mean diameter of $93 \pm 4 \AA( \pm \operatorname{SEM}) n=197$. Negative stain electron microscopy of mesenteric lymph HDL from four rats however, showed discoidal HDL particles in all preparations examined (Fig. $1 b, c$ ). Discoidal HDL particles tended to form rouleaux and were more prominent in lymph fractions collected with DTNB as compared to fractions collected without DTNB. To determine the dimensions of these discoidal structures, particle measurements were carried out only on clearly apparent discoidal structures. The dimensions of discoidal HDL particles, only measured from disks on edge, was $190 \pm 55 \pm 1 \AA( \pm$ SEM $) n=166$, with the diameter of $90 \%>160 \AA$. In contrast, $90 \%$ of serum HDL particles was $<160 \AA$ in diameter. It is apparent that only a portion of the particles visualized in fasting or fatty lymph (Fig. $1 b, c)$ can clearly be identified as discoidal. To determine the size distribution of particles in lymph HDL, particle measurements were carried out on all particles in these fractions. These measurements were performed on randomly selected fields from micrographs of fasting and fatty lymph HDL. In fasting lymph HDL, $50 \%$ of all particles was $(n=378)>160 \AA$ in diameter. While in fatty lymph, $35 \%$ of particles counted $(n=200)$ was $>160 \AA$ in diameter (Fig. 2). These results suggest that the largest spherical particles may be disks seen en face. Therefore, discoidal HDL may represent a major component of lymph HDL, especially in the fasting state.

Lipid analysis. Lipid analysis of HDL was carried out to determine whether additional information could be obtained about the relative contribution of discoid HDL to lymph HDL. Lymph HDL, compared to serum HDL, was remarkable for its increased ratio of polar to nonpolar lipids (Table I). Thus, the ratio of phospholipids/cholesterol esters (PL/CE) was significantly increased in fasting and fatty lymph (4.5 and 2.0 , respectively), when compared to serum HDL (0.9) 

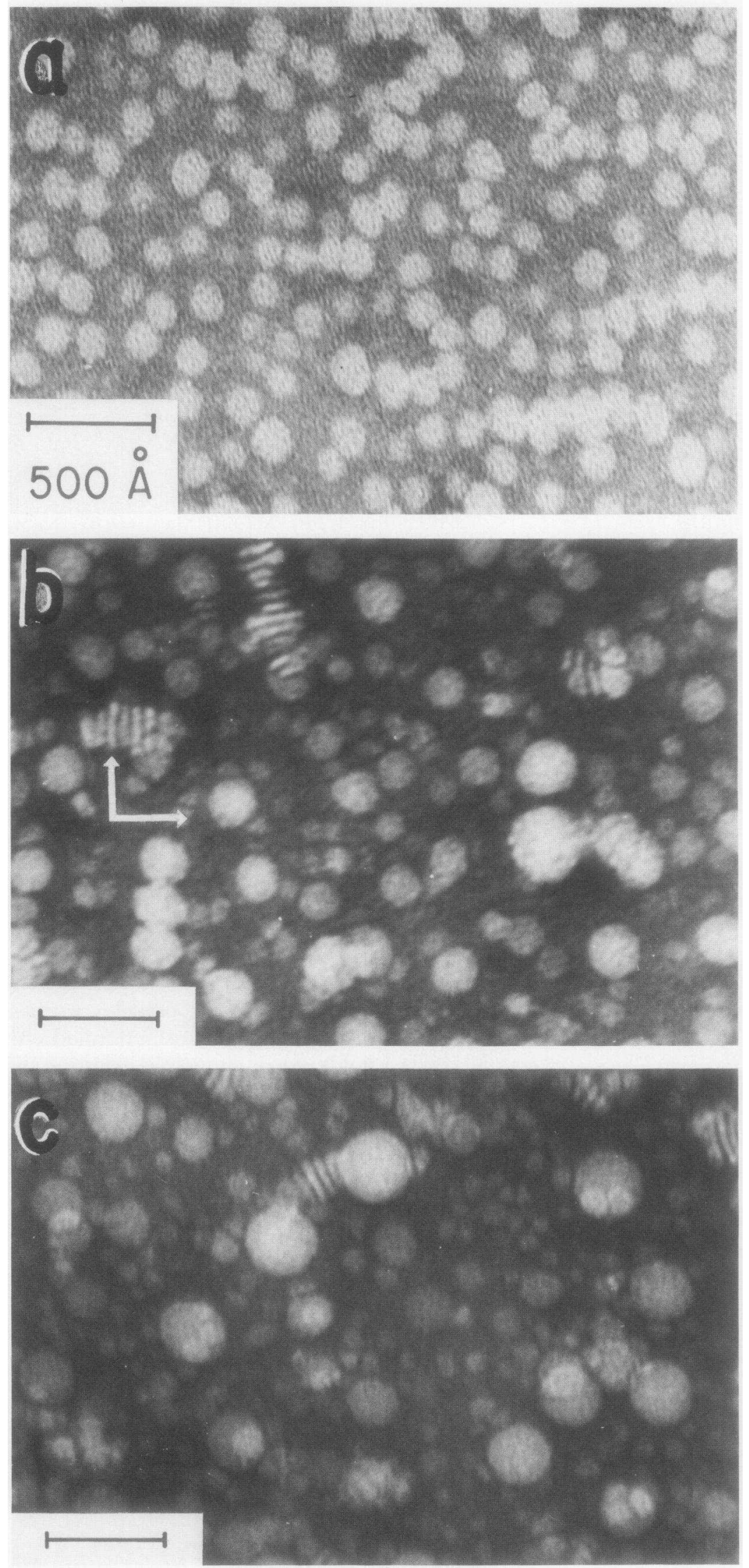


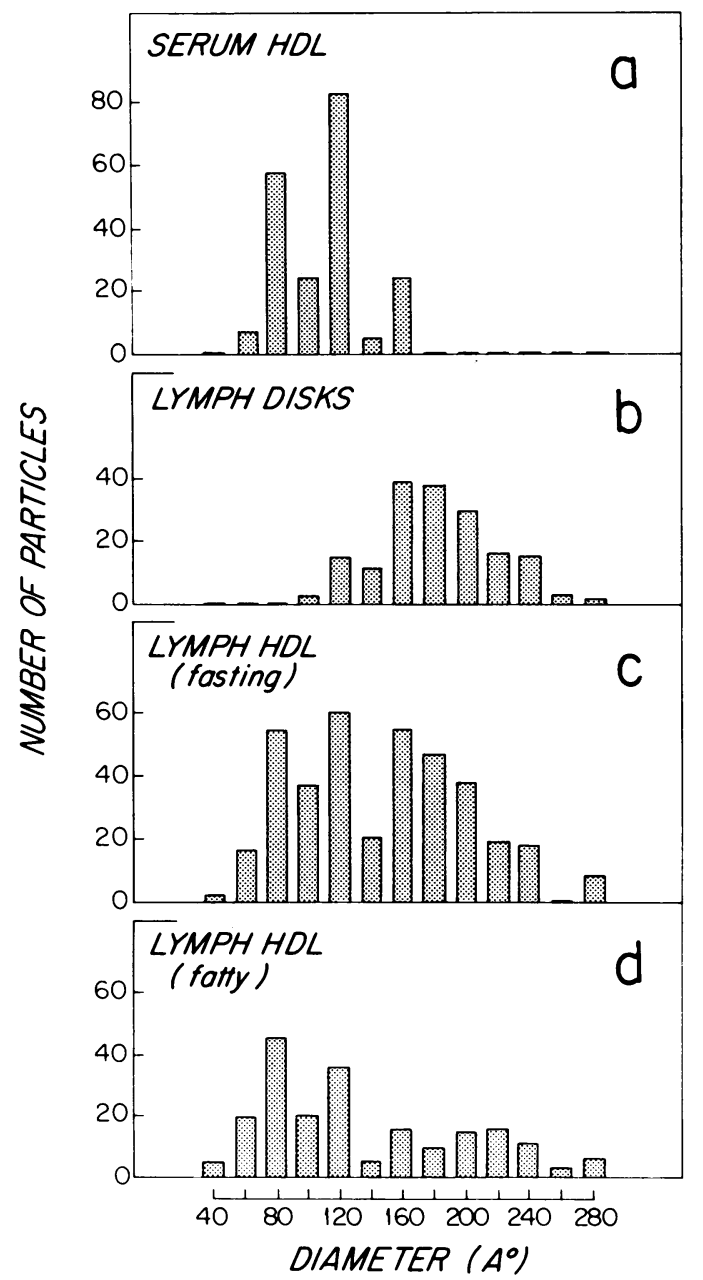

Figure 2 Size distribution of serum and lymph HDL particles from negative stain electron micrographs. Particle diameters were measured from unselected fields (Methods). $(a, b)$ HDL from serum $(93 \pm 4 \AA$ diameter, $n=197)$ and obvious discoid HDL particles $(190 \pm 3 \times 55 \pm 1 \AA, n=166)$ from lymph were sized. $90 \%$ of disks seen on edge were $>160$ $\AA$. $(c, d)$ HDL particles from fasting $(n=378)$ and fatty lymph $(n=200) .50 \%$ of HDL particles in basal lymph and $35 \%$ of HDL particles in fatty lymph were $>160 \AA$. $(n=$ number of particles counted)

(Table I). The PL/CE ratio of fasting lymph was significantly greater than fatty lymph, and was greater in samples collected in the presence of DTNB. The lipid to protein ratio was increased in mesenteric lymph HDL as compared to serum HDL. These compositional data reflect the presence in lymph of HDL which are deficient in cholesterol ester. This is most apparent in the HDL fraction isolated from fasting lymph which was collected with DTNB.

Assuming that lymph HDL is a mixture of discoidal HDL containing only polar lipids and protein, and the composition of spherical HDL resembles that of serum HDL, the compositional data indicate that $\cong 50 \%$ of the particles in fasting lymph and $30 \%$ of particles in fatty lymph are discoidal HDL. ${ }^{2}$

Apoprotein composition. Serum and lymph HDL, isolated in the presence of DTNB, were purified, delipidated, and then the apoproteins were subjected to sodium dodecyl sulfate gel electrophoresis (Fig. 3). Two major apoproteins were seen in all samples. The major apoprotein band of all samples was apoA-I (mol wt 25,000$)(5)$. The other prominent apoprotein band has a mol wt $\sim 35,000$ and corresponded to the arginine-rich protein (ARP) (15). The apoprotein bands were quantitated by planimetry of densitometric scans (Table II). Basal lymph HDL was enriched in apoA-I $(\mathrm{A}-\mathrm{I} / \mathrm{ARP}=3.2)$ as compared to serum HDL (A-I/ $\mathrm{ARP}=1.1 P<0.0025)$, whereas the apoprotein composition of HDL from fatty lymph (A-I/ARP $=1.3$ ) more closely resembled serum HDL, suggesting the presence of HDL transferred from serum.

When isolated without the addition of DTNB, the apoprotein composition of HDL from fasting lymph (A-I/ARP = 3.4) and HDL from fatty lymph (A-I/ ARP $=1.7$ ) was similar to HDL isolated in the presence of the LCAT inhibitor.

In addition, high molecular weight bands were noted in the gels of each HDL sample (Fig. 3). Similar bands have previously been described in serum HDL (15) and liver perfusate HDL $(16,17)$. Recent evidence suggests that a portion of this protein may represent apoB associated with the HDL fraction (18).

\section{DISCUSSION}

The results of the present study provide direct evidence that the intestine secretes HDL into mesenteric lymph as discoidal particles. Negative stain electron microscopy of rat mesenteric lymph revealed discoid particles $(190 \pm 3 \times 55 \pm 1 \AA)$ which tended to form rouleaux. These dimensions together with the high PL/CE ratio

${ }^{2}$ These results were calculated from the volume of a disk with the dimensions of $190 \times 55 \AA$, assuming an identical partial specific volume for discoidal and spherical HDL.

FIGURE 1 Negative stain electron microscopy of HDL $(d=1.075-1.21 \mathrm{gm} / \mathrm{ml})$ from rat serum and mesenteric lymph. (a) Serum HDL, with added DTNB: A uniform population of spherical particles is present. (b) Fasting lymph HDL with added DTNB: Note the disks on edge which tend to form rouleaux. In addition, particles of similar diameter to the disks are present (arrows). Spherical particles similar to serum HDL are also present. $(c)$ HDL from fatty lymph with added DTNB: Disks on edge are also seen. 
TABLE I

Composition of Mesenteric Lymph HDL*

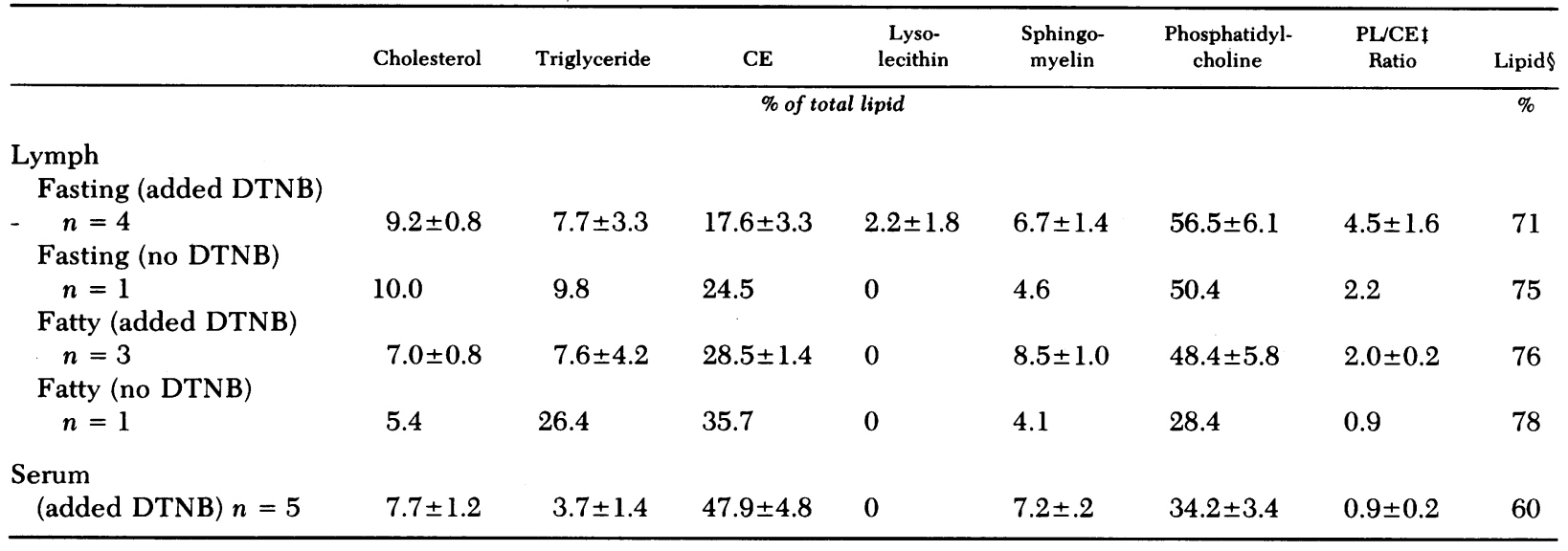

* All values are mean $\pm \mathrm{SEM}$.

$\ddagger P$ values for $\mathrm{P} / \mathrm{CE}$ ratios (from Student's $t$ test for unpaired observations). Fasting vs. fatty $P<0.05$; fasting vs. serum $P<0.025$; fatty vs. serum $P<0.01$.

$\$$ Lipid/lipid + protein $\times 100 \%$.

of the HDL fraction confirm that the structure of these HDL particles is a phospholipid bilayer. The discoidal shape of nascent intestinal HDL resembles recombi-

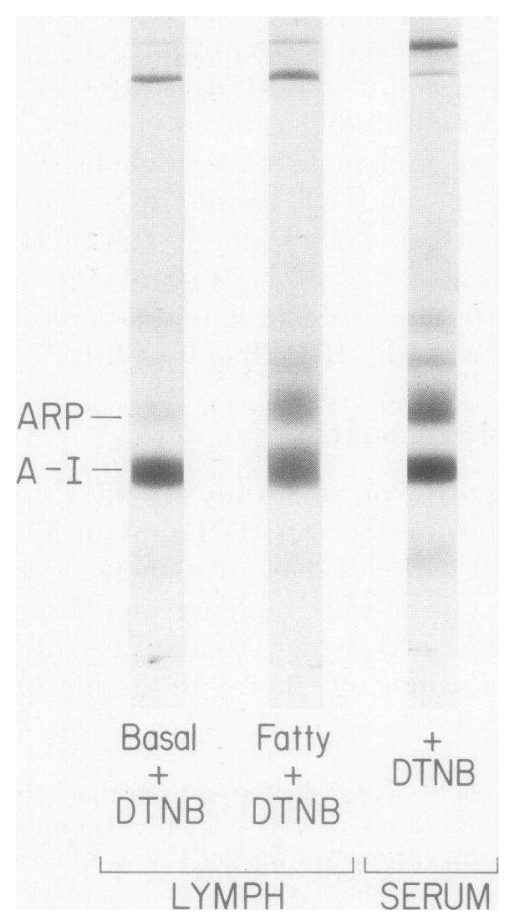

FIGURE 3 Sodium dodecyl sulfate polyacrylamide gels of lymph and serum HDL. HDL was isolated from basal and fatty lymph and serum in the presence of DTNB. The major bands seen correspond to the ARP and apoA-I. HDL from fasting basal lymph contains mainly apoA-I, while fatty lymph HDL more closely resembles serum HDL. nant HDL (19) in which the apoprotein forms an annulus around the edge of the phospholipid bilayer (20). Discoidal HDL were not seen in the serum where spherical HDL particles (diameter $93 \pm 4 \AA$ ) were uniformly seen. This eliminated the possibility that discoidal HDL was filtered from plasma into lymph. Previous evidence that the intestine secreted HDL was limited to studies in which the intestine was shown to incorporate labeled amino acids into HDL apoproteins $(3,4)$. However, the interpretation of these results is complicated by the finding that lipids and apoproteins readily transfer among lipoproteins (21).

The nascent form of rat hepatic HDL is discoid (8) and human. HDL seems to be secreted in a similar form, for the HDL in the serum of people with genetic LCAT deficiency (22), alcoholic hepatitis (23), and cholestasis

TABLE II

Apoprotein Composition of $H D L$

\begin{tabular}{ccc}
\hline Source of HDL & Ratio A-I/ARP & $P$ \\
\hline $\begin{array}{c}\text { Serum }(n=4) \\
\text { + DTNB }\end{array}$ & $1.1 \pm 0.04$ & \\
$\begin{array}{c}\text { Basal lymph } \\
\text { + DTNB }(n=6)\end{array}$ & $3.2 \pm 0.3$ & $<0.0025$ \\
$\begin{array}{l}\text { Fatty lymph } \\
\text { + DTNB }(n=3)\end{array}$ & $1.3 \pm 0.2$ & $>0.05$ \\
\hline
\end{tabular}

Sodium dodecyl sulfate acrylamide gel electrophoresis was performed on delipidated HDL apoproteins isolated from serum and lymph with the addition of DTNB. Gels were scanned densitometrically and the protein bands quantitated by planimetry. Values are the ratio of the areas of each protein band (mean \pm SEM). 
(24) has been noted to be discoid. The discoidal HDL particle is probably converted to a spherical particle by the action of the enzyme $\operatorname{LCAT}(8,19)$. CE formed from surface phospholipid and free cholesterol, accumulates within the bilayer forming a spherical HDL particle of smaller size, rich in cholesterol ester and deficient in surface phospholipids. Our findings of fewer discoidal HDL particles in the absence of DTNB as well as an increase in cholesterol ester, suggest that LCAT may have a similar action on mesenteric lymph HDL.

Mesenteric lymph, obtained in the basal state and after lipid absorption, contained discoidal nascent and spherical HDL. To quantitate the proportion of HDL found in mesenteric lymph secreted by the intestine, as opposed to the HDL present as a result of filtration from the plasma compartment, we analyzed the data of the lipid composition presented in Table I as well as the size of the HDL particles shown in Fig. 2. We estimate that $50 \%$ of the HDL particles are discoid in fasting mesenteric lymph. It is known that the capillary filtration of plasma increases during lipid absorption, and leads to increased lymph flow and plasma protein secretion into lymph (25). Therefore, the increased proportion of spherical HDL seen in fatty lymph could be explained by a greater transfer of plasma HDL to lymph during lipid absorption. In addition, LCAT activity increases in mesenteric lymph after lipid feeding (9) and could account for the presence of greater numbers of spherical particles enriched in cholesterol esters. Since DTNB was added during the collection of lymph, modification of nascent disks could have occurred before collection. Thus, our data may have underestimated the proportion of HDL secreted from the intestine as discoidal particles.

The major differences between nascent intestinal HDL and nascent hepatic HDL are the apoprotein composition and $\mathrm{PL} / \mathrm{cholesterol} \mathrm{ratio.} \mathrm{Hamilton} \mathrm{et} \mathrm{al.}$ (8) and Marsh (17) reported that ARP is the major apoprotein of hepatic HDL although we have found apoA-I to be the major apoprotein of intestinal HDL. HDL from fatty lymph had more ARP compared to fasting samples (A-I/ARP 1.3 of. 3.2 Table II) and reflects the increased proportion of filtered serum HDL. Although ultracentrifugation has been shown to result in the dissociation of apoA-I (26) and ARP (27) from lipoproteins, it is unlikely to explain the findings in the present study because all lymph and plasma samples were treated identically. Finding that intestinal HDL is rich in apoA-I underscores the importance of the intestine as a source of apoA-I. Rat mesenteric lymph chylomicrons, very low density lipoproteins (5) and nascent intestinal HDL are enriched in apoA-I. In contrast, it appears that the isolated perfused liver has only a limited capacity to synthesize and secrete apoA-I compared with ARP $(28,29)$. The apoprotein composition of lymph HDL was independent of the presence of
DTNB, suggesting that in mesenteric lymph the LCAT reaction may not be intimately related to apoprotein transfer as suggested with hepatic HDL (8).

The present findings demonstrate that the intestine produces HDL as a phospholipid bilayer rich in apoA-I and may therefore be an important source of plasma HDL. It has been suggested by Glomset (30) that HDL has a major role in the removal of cholesterol from tissues and transportation of cholesterol to the liver for catabolism and excretion. Thus, the finding that intestinal HDL (PL/cholesterol $=7$ ) is more deficient in cholesterol than nascent hepatic HDL (PL/ cholesterol $=3)(8)$ is of potential importance. It would appear that nascent intestinal HDL is an ideal acceptor for cholesterol because it is more deficient in cholesterol than other lipoproteins and cell membranes. Therefore, intestinal HDL may be important in cholesterol homeostasis, providing a reservoir for tissue and lipoprotein cholesterol and ultimately a vehicle of cholesterol removal.

\section{ACKNOWLEDGMENT}

This research was supported by grant AM 18911 from the National Institutes of Health.

\section{REFERENCES}

1. Glickman, R. M., and K. Kirsch. 1973. Lymph chylomicrons formation during the inhibition of protein synthesis. J. Clin. Invest. 52: 2910-2920.

2. Ockner, R. K., F. B. Hughes, and K. J. Isselbacher. 1969. Very low density lipoproteins in intestinal lymph. Origin, composition and role in lipid transport in the fasting state. J. Clin. Invest. 48: 2079-2088.

3. Roheim, P. S., L. I. Gidez, and H. A. Eder. 1966. Extrahepatic synthesis of lipoproteins of plasma and chyle. Role of the intestine. J. Clin. Invest. 45: 297-300.

4. Windmueller, H. G., and A. E. Spaeth. 1972. Fat transport and lymph and plasma lipoprotein biosynthesis by isolated intestine. J. Lipid Res. 13: 92-105.

5. Glickman, R. M., and P. H. R. Green. 1977. The intestine as a source of apolipoprotein A-I. Proc. Natl. Acad. Sci. U. S. A. 74: 2569-2573.

6. Courtice, F. C., and B. Morris. 1955. The exchange of lipids between plasma and lymph of animals. Q. J. Exp. Physiol. Cogn. Med. Sci. 40: 138-148.

7. Hamilton, R. L. 1972. Synthesis and secretion of plasma lipoproteins. Adv. Exp. Med. Biol. 26: 7-24.

8. Hamilton, R. L., M. C. Williams, C. J. Fielding, and R. J. Havel. 1976. Discoidal bilayer structure of nascent high density lipoproteins from perfused rat liver. J. Clin. Invest. 58: 667-680.

9. Bennett-Clark, S., and K. R. Norum. 1977. The lecithincholesterol acyltransferase activity of rat intestinal lymph. J. Lipid Res. 18: 293-300.

10. Brown, W. V., R. I. Levy, and D. S. Fredrickson. 1969. Studies of the proteins in human plasma very low density lipoproteins. J. Biol. Chem. 244: 5687-5694.

11. Folch, J., M. Lees, and G. H. Sloane Stanley. 1951. A simple method for the isolation and purification of total lipids from animal tissues. J. Biol. Chem. 226: 497-509.

12. Downing, D. T. 1968. Photodensitometry in the thin-layer 
chromatographic analysis of neutral lipids.J. Chromatogr. 38: $91-99$.

13. Katz, S. S., G. G. Shipley, and D. M. Small. 1976. Physical chemistry of the lipids of human atherosclerotic lesions.J. Clin. Invest. 58: 200-211.

14. Lowry, O. M., N. J. Rosebrough, A. K. Farr, and R. J. Randall. 1951. Protein measurement with the Folin phenol reagent. J. Biol. Chem. 193: 265-275.

15. Swaney, J. B., H. Reese, and H. A. Eder. 1974. Polypeptide composition of rat high density lipoprotein: characterization by SDS-gel electrophoresis. Biochem. Biophys. Res. Commun. 59: 513-519.

16. Noel, S. D., and D. Rubenstein. 1974. Secretion of apolipoproteins in very low density and high density lipoproteins by perfused rat liver. J. Lipid Res. 15: $301-308$.

17. Marsh, J. B. 1976. Apoproteins of the lipoproteins in a non-recirculating perfusate of rat liver. J. Lipid Res. 17: 85-90.

18. Fainaru, M., T. E. Felker, R. L. Hamilton, and R. J. Havel. 1977. Evidence that a separate particle containing $B$ apoprotein is present in high density lipoprotein from perfused rat liver. Metab. Clin. Exp. 26: 999-1004.

19. Forte, T. M., A. V. Nichols, E. C. Gong, S. Lux, and R. I. Levy. 1971. Electron microscopic study on reassembly of plasma high density apoprotein with various lipids. Biochim. Biophys. Acta. 248: 318-386.

20. Tall, A. R., D. M. Small, R. J. Dekelbaum, and G. G. Shipley. 1977. Structure and thermodynamic properties of high density lipoprotein recombinents. J. Biol. Chem. 52: 4701-4712.

21. Havel, R. J., J. P. Kane, and M. L. Kashyap. 1973. Interchange of apolipoproteins between chylomicrons and high density lipoproteins during alimentary lipemia in man. J. Clin. Invest. 52: 32-38.

22. Forte, T., K. R. Norum, S. A. Glomset, and A. V. Nichols. 1971. Plasma lipoproteins in familial lecithin: cholesterol acyltransferase deficiency. Structure of low and high density lipoproteins as revealed by electron microscopy. $J$. Clin. Invest. 50: 1141-1148.

23. Sabesin, S. M., H. L. Hawkins, K. Kuiken, and J. B. Ragland. 1977. Abnormal plasma lipoproteins and lecithin-cholesterol acyltransferase deficiency in alcoholic liver disease. Gastroenterology. 72: 510-518.

24. Hamilton, R. L., R. J. Havel, and M. C. Williams. 1974 Lipid bilayer structure of plasma lipoproteins in cholestasis. Fed. Proc. 33: 351. (Abstr.)

25. Wollin, A., and L. B. Jaques. 1973. Plasma protein escape from the intestinal circulation into lymphatic during fat absorption. Proc. Soc. Exp. Biol. Med. 142: 1114-1117.

26. Fainaru, M., R. J. Havel, and T. E. Felker. 1976. Radioimmunoassay of apolipoprotein A-I of rat serum. Biochim. Biophys. Acta. 446: 56-68.

27. Mahley, R. W., and K. S. Holcombe. 1977. Alterations of the plasma lipoproteins and apoproteins following cholesterol feeding in the rat. J. Lipid Res. 18: 314-324.

28. Felker, T. E., M. Fainaru, R. L. Hamilton, and R. J. Havel. 1976. Secretion of apolipoproteins (A-I and ARP) by the isolated perfused rat liver. Circulation. 54(Suppl. II): 92. (Abstr.)

29. Schonfeld, G., A. R. Brown, C. E. Bell, Jr., and D. H Alpers. 1977. Apo-(lipo)protein A-I production by the rat small intestine. Gastroenterology. 72: A-104, 1127.

30. Glomset, J. A. 1968. The plasma lecithin: cholesterol acyltransferase reaction. J. Lipid Res. 9: 155-167. 